\title{
Clinicians as managers
}

\author{
Z. Latif
}

Received: 5 May 2009/ Accepted: 13 September 2010/Published online: 26 September 2010

(C) Royal Academy of Medicine in Ireland 2010

\section{Sir,}

Healthcare has become a complex business and far from a simple exercise, which requires expertise in healthcare management to operate efficiently, especially when resources are limited. Due to increased reliance on economic efficiency, quality of care, consumer involvement, accountability, and scientific methods of service delivery, it is impossible for clinicians to keep themselves away from management issues. The role of a consultant is evolving with responsibilities as a clinician, teacher, manager and leader.

Most doctors are reluctant to participate in management due to lack of time, poor involvement in management by the managers, lack of understanding of management culture, and lack of opportunities to get training in management in spite of a strong inner desire to be involved which leaves them frustrated at times. Most clinicians are content and rewarded by their clinical work. There are few who accept challenge but want to retain credibility with fellow clinicians by retaining an active clinician's role as well which makes it further difficult for them to fulfil two roles. On the other side, it is difficult in our current healthcare structure to make a clinician's role meaningful with realistic involvement in management of healthcare delivery. Ross states that "Power without responsibility is dangerous, but responsibility without power is frustrating".
In spite of lot of government initiatives, doctors are not sure what role is expected from them, whether it should be, "doctors as managers", "doctors in management", or "doctors and management". Due to current competitive environment to get higher specialist training schemes and substantive consultant posts, most doctors do different types of courses ranging from a day course to masters in healthcare management but experiencing difficulties in finding structure which facilitate their involvement in management particularly as non-consultant hospital doctors. At present in Republic of Ireland, we are going through a process of selection or recruitment of clinical directors in different specialities and regions without clarifying role, responsibilities and powers of this new post. It is also mentioned that if all colleagues unanimously select a consultant as a clinical director, then there will be no interview which in my opinion undermines the formal training, experience and management capabilities of the person. In my opinion it is important to facilitate a participative relationship between clinician's and managers which starts from a very early stage as a non-consultant hospital doctor and increases as he/she progresses in his/her clinical career which include management training in their organisational context which will result in new generation of clinicians as managers.
Z. Latif $(\square)$

St Davnet's Hospital, Monaghan, Ireland

e-mail: drzahidlatif@gmail.com 